\title{
Quantifications for multi-mode entanglement
}

\author{
Mehmet Emre Tasgin ${ }^{1, *}$ and M. Suhail Zubairy ${ }^{2}$ \\ ${ }^{1}$ Institute of Nuclear Sciences, Hacettepe University, 06800, Ankara, Turkey \\ ${ }^{2}$ Institute of Quantum Studies and Department of Physics, \\ Texas A \& $M$ University, College Station, TX 77 843-4242, USA
}

(Dated: May 7, 2019)

\begin{abstract}
We introduce two independent quantifications for 3-mode and 4-mode entanglement. We investigate the conversion of one type of nonclassicality, i.e. single-mode nonclassicality, into another type of nonclassicality, i.e. multi-mode entanglement, in beam-splitters. We observe parallel behavior of the two quantifications. The methods can be generalized to the quantification of any multi-mode entanglement.
\end{abstract}

Multi-mode continuous variable entanglement is a key element for quantum information processing. Quantum teleportation among multiple parties [1], quantum computation with clusters [2, 3], e.g. quantum networks, [4] and quantum internet [5], all, necessitate the presence of multi-mode entanglement. Multi-mode entanglement can be generated using several number of beam-splitters which converts the single-mode nonclassicality (SMNc), e.g. quadrature squeezing, of input beam(s) into multimode entanglement at the outputs of the beam splitters [6]. Hence, mechanism of the conversion of nonclassicality into multi-mode entanglement, via beam splitters, is important for distribution of the entanglement among multi-parties used in quantum information.

One can notice that quantum nonclassicality is like "energy". That is, it can be converted into different forms. For instance, single-mode nonclassicality $(\mathrm{SMNc})$, two-mode entanglement (TME) and manyparticle entanglement (MPE) are different forms nonclassicality (Nc) and can be converted to each other via linear interactions. [1] A quadrature-squeezed light (SMNc) can transfer its squeezing to an ensemble of atoms [8[10] as spin-squeezing [11]. This creates MPE [7, 12] in the ensemble. Similarly, interaction of an ensemble with two entangled beams (TME) also creates MPE in the ensemble [13, 14]. It is also possible to convert the nonclassicality of a single-mode, e.g. quadrature-squeezing, into two-mode entanglement at the output of a beamsplitter (BS) [15, 16]. Generation of TME in a BS necessitates a nonclassical input light 15.

Recent works [17-20] show that there appears a conservation-like relation between the generated TME and the remaining SMNc in a BS. A BS cannot convert all of the nonclassicality of the input beam into TME at the output. There still remains some SMNc in the two output modes [17]. However, the total SMNc decreases with respect to the input one. The form of the analytical expressions for the maximum TME extracted at the BS output, $E_{\mathcal{N}}=\max \left\{0,-\frac{1}{2} \log _{2}\left(\lambda_{1, \mathrm{sm}}^{(\mathrm{in})} \lambda_{2, \mathrm{sm}}^{(\mathrm{in})}\right)\right\}[21$, 22],

\footnotetext{
* metasgin@hacettepe.edu.tr

1 Also the criteria witnessing them can be transformed into each other [7.
}

suggests us to quantify the SMNc in terms of a noisearea [17, $\Omega=\lambda_{1, \mathrm{sm}} \lambda_{2, \mathrm{sm}}$. Here, $\lambda_{i, \mathrm{sm}}$ is the minimum noise of the $i$ th beam input into the BS, where $\lambda_{i, \mathrm{sm}}<1$ implies the presence of squeezing in the $i$ th beam. For mixing with vacuum or a coherent state, one of the modes becomes $\lambda_{1, \mathrm{sm}}=1$.

As an illuminating example: if a squeezed beam is mixed with a thermal noise [21] at the input channels of a BS, the output modes are entangled only if the noisearea $\Omega=\lambda_{\mathrm{sqz}}(1+2 \bar{n})<1$, where $\lambda_{\mathrm{sqz}}<1$ is the reduced noise of the squeezed beam and $\bar{n}$ is the mean number of photons in the thermal noise. Also Ref. [22] shows that the maximum amount of entanglement extractable at the BS output is $\left.E_{\mathcal{N}}=-\frac{1}{2} \log _{2}\left(2 \nu_{-}\right)=-\frac{1}{2} \log _{2}\left(\lambda_{1, \mathrm{sm}}^{(\mathrm{in})} \lambda_{2, \mathrm{sm}}^{(\mathrm{in})}\right)\right\}$ if any two Gaussian states are mixed in the BS input. Here, one can observe that the smallest symplectic eigenvalue of an inseparable system [23, 24] becomes $\mu=2 \nu_{-}=\lambda_{1, \mathrm{sm}}^{(\mathrm{in})} \lambda_{2, \mathrm{sm}}^{(\mathrm{in})}$, which is actually the input noisearea. In Ref. [17, we further show that the output TME is proportional to the change (increase) in the noisearea $S_{N}=\log _{2}\left(\lambda_{1, \mathrm{sm}}^{\text {(out) }} \lambda_{2, \mathrm{sm}}^{\text {(out) }}\right)-\log _{2}\left(\lambda_{1, \mathrm{sm}}^{\text {(in) }} \lambda_{2, \mathrm{sm}}^{\text {(in) }}\right)$ of the out beams with respect to the input beams. A geometric demonstration of this $\mathrm{SMNc} \rightarrow \mathrm{TME}$ swap can be found in Fig. 1 of Ref. [25]. More interestingly, well-known TME criteria, like Duan-Giedke-Cirac-Zoller [26] and HilleryZubairy [27, actually do search for a noise-area below unity in BS-like rotations 25].

In the present work, we investigate the swapping of single-mode nonclassicality into multi-mode entanglement (MME). In particular, we study three-mode entanglement (3ME) and four-mode entanglement (4ME) after two (Fig. 1) and three beam-splitters (Fig. 3), respectively. We define the remaining SM nonclassicalities in the 3 modes (or 4 modes) as a noise-volume (or a $4 \mathrm{D}$ noise-volume), $\Omega=\lambda_{1, \mathrm{sm}}^{\text {(out) }} \lambda_{2, \mathrm{sm}}^{\text {(out) }} \lambda_{3, \mathrm{sm}}^{\text {(out) }}$. Here, $\tau_{i}^{\text {(out) }}=1-\lambda_{i \text {, sm }}^{\text {(out) }}$ is the degree of the remaining SMNc, i.e. the nonclassical depth [28], of each mode after the BSs. It is calculated by wiping out the entanglement (correlations) between the modes [17. We show that the SMNc decreases (noise-volume $\Omega$ increases) after the BSs while the the $3 \mathrm{ME}$ increases.

In the quantification of $3 \mathrm{ME}$ we use two different (independent) approaches. (i) We multiply the three noises as- 
sociated with the symplectic eigenvalues $\left(\nu_{1}, \nu_{2}, \nu_{3}\right)$ of the partial transposed system. $\nu_{1}$, for instance, is the smallest symplectic eigenvalue of the 3-mode system when the 1st mode is partial transposed [23, 24]. That is, smaller values of the eigenvalue compared to unity, i.e. $2 \nu_{1}<1$, imply stronger entanglement of the 1st mode with the system composed of $(2 \mathrm{nd}+3 \mathrm{rd})$ modes [29. Similarly, $2 \nu_{2}<1$ refers to the inseparability of the 2 nd mode from the system of $(1 \mathrm{st}+3 \mathrm{rd})$ modes. Hence, $\mu=2 \nu_{1} 2 \nu_{2} 2 \nu_{3}$ refers to a kind of 3-mode entanglement strength, where $2 \nu_{i}>1$ implies the absence of a genuine 3-mode entanglement.

(ii) Second, we use an alternative method given in Sec. II.3.2 of Ref. [25]. The idea is very simple: Nonclassical depth $\tau$ quantifies the whole nonclassicality, i.e. $\mathrm{SMNc}+$ entanglement, in a multi-mode system [22]. If we remove the unconverted (unused) SM nonclassicalities (after the BSs) from the noise-matrix, then the remaining nonclassicality is due to the entanglement only ${ }^{2}$. Nonclassicality of a single-mode state can be determined by introducing a Gaussian filter function transformation on the Glauber-Sudarshan $P$-function, i.e. $P(\alpha, \tau)=$ $\int d^{2} \alpha^{\prime} \exp \left\{-\left|\alpha-\alpha^{\prime}\right|^{2} / \tau\right\} P\left(\alpha^{\prime}\right) / \tau \pi$. So that, the new $P$-function is non-negative [28]. This corresponds to injecting noise which destroys the nonclassicality [30]. A similar method can be used also to determine the nonclassicality of a multi-mode system 22. Here, in difference to Ref. 22, we introduce different $\tau$ 's for each mode. This way, we prevent the injection of unnecessary noise $(\tau)$ by constraining $\tau_{1}=\tau_{2}=\tau_{3}=\tau$.

We emphasize that, our aim, in this short manuscript, is to introduce the basics of two possible quantifications for the multi-mode entanglement (MME). We do not aim to present a detailed analysis on MME, but we suffice with demonstrating that SMNc, quantified as a noisevolume, is converted into MME.

\section{3-mode entanglement}

We study the system depicted in Fig. 1. A singlemode (SM) nonclassical state $\hat{a}$ is mixed with vacuum noise in a BS with two output states $\hat{a}_{1}$ and $\hat{a}_{2}$. One of the output modes, $\hat{a}_{2}$, is input to a second $\mathrm{BS}$, mixed with vacuum, resulting two new output modes $\hat{b}_{1}$ and $\hat{b}_{2}$. We examine the 3 -mode entanglement (3ME) of $\hat{a}_{1}, \hat{b}_{1}$, and $\hat{b}_{2}$ modes.

In Fig. $2 \mathrm{a}$ and Fig. $2 \mathrm{p}$, we examine the $3 \mathrm{ME}$ of the $\hat{a}_{1}$, $\hat{b}_{1}, \hat{b}_{2}$ modes using the two methods, $(i)$ and (ii), respectively. We observe a similar behavior for the two quantifications. In Fig. 22, one can observe that the SM nonclassicalities remaining in the $\hat{a}_{1}, \hat{b}_{1}, \hat{b}_{2}$ modes decreases (noise-volume increases), while the $3 \mathrm{ME}$ increases. In all Fig. 2a-2r, smaller $\mu, \eta$ and $\mathcal{N}$ imply stronger $3 \mathrm{ME}$

2 In Ref. [17, we do the reverse. We remove the correlations in the noise matrix and examine the remaining SM nonclassicalitites.

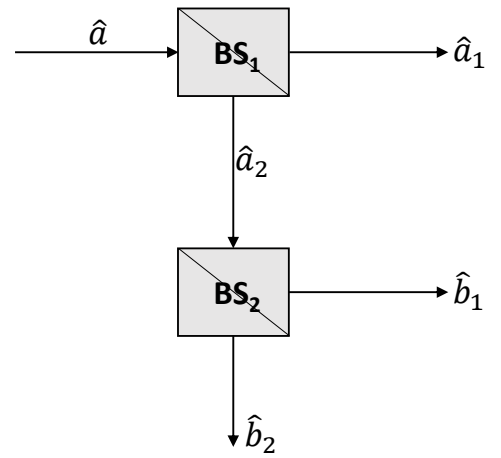

FIG. 1. Creation of 3-mode entanglement (3ME) using 2 beam-splitters. We examine the $3 \mathrm{ME}$ of $\hat{a}_{1}, \hat{b}_{1}, \hat{b}_{2}$ modes. We also investigate the conversion of the single-mode nonclassicality into 3ME, see Fig. 2]
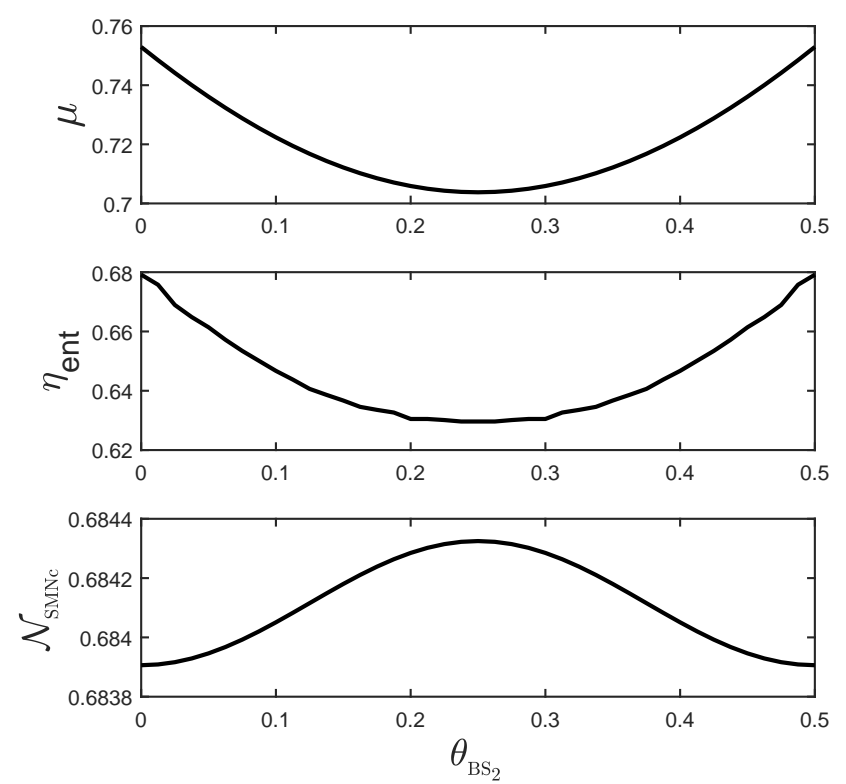

FIG. 2. Conversion of SMNc into 3-mode entanglement (3ME). (a) Quantification of 3ME via symplectic eigenvalues $\mu=2 \nu_{1} 2 \nu_{2} 2 \nu_{3}$ where $2 \nu_{i}$ is obtained by the partial transposition of the $i$ th mode. See method (i) in the text. (b) Quantification of the entanglement by wiping out the singlemode nonclassicalities from the noise-matrix, where the remaining nonclassicality refers to the entanglement only. See method (ii) in the text. (c) The remaining single-mode nonclassicality $(\mathrm{SMNc})$ in the output modes when the correlations are wiped out, in stead of the SMNc. Smaller $\mu, \eta_{\text {ent }}, \mathcal{N}<1$ implies a stronger entanglement/nonclassicality strength.

or SMNc. The first BS fed with a nonclassical light of squeezing parameter 31] $r=0.1$. We fix the angle of the first $\mathrm{BS}$ to $\theta_{\mathrm{BS}_{1}}=\pi / 4$ and vary the angle of the second BS $\theta_{\mathrm{BS}_{2}}$.A similar behavior is obtained for varying $\theta_{\mathrm{BS}_{1}}$ with a fixed $\theta_{\mathrm{BS}_{2}}$.

$\mu, \eta_{\text {ent }}$ and $\mathcal{N}$ are calculated from the 3 mode noise 
matrix as follows.

Calculation of $\boldsymbol{\mu}$.- We compose the $6 \times 6$ noise-matrix $V_{i j}=\left\langle\hat{\xi}_{i} \hat{\xi}_{j}+\hat{\xi}_{j} \hat{\xi}_{i}\right\rangle / 2-\left\langle\hat{\xi}_{i}\right\rangle\left\langle\hat{\xi}_{j}\right\rangle$ for the 3 mode system in the real representation [32] by introducing the operator $\hat{\xi}=\left[\hat{x}_{1}, \hat{p}_{1}, \hat{x}_{2}, \hat{p}_{2}, \hat{x}_{3}, \hat{p}_{3}\right]$, where $\hat{x}_{i}=$ $\left(\hat{c}_{i}^{\dagger}+\hat{c}_{i}\right) / \sqrt{2}$ and $\hat{p}_{i}=i\left(\hat{c}_{i}^{\dagger}-\hat{c}_{i}\right) / \sqrt{2}$ are obtained using $\hat{c}_{i}=\hat{a}_{1}, \hat{b}_{1}, \hat{b}_{2}$, respectively. Partial transpose (PT) operation, e.g., on the 1st mode is equivalent to $\hat{p}_{1} \rightarrow-\hat{p}_{1}$ in the noise-matrix [33]. If the 1st mode is separable from the system of $(2+3)$ modes, symplectic eigenvalues of the partial transposed noise-matrix must be all larger than $1 / 2$ [23, 24]. (So, smallest eigenvalue $\nu_{1}$ also satisfies $2 \nu_{1}>1$.) The symplectic eigenvalues of the partial transposed noise-matrix can alternatively be calculated as eig $\left[i \chi_{1} V\right]$ where $\chi_{1}$ is the $6 \times 6$ matrix $\chi_{1}=\left[-J, 0_{2 \times 2}, 0_{2 \times 2} ; 0_{2 \times 2}, J, 0_{2 \times 2} ; 0_{2 \times 2}, 0_{2 \times 2}, J\right]$ with $0_{2 \times 2}$ is $2 \times 2$ matrix of zeros and $J=[0,1 ;-1,0]$. For $\chi_{2}$ and $\chi_{3}$ the "-" sing must be in the second and third $J$, respectively. eig $\left[\mathrm{i} \chi_{1} \mathrm{~V}\right]$ yields only a single eigenvalue with $2 \nu_{1}<1$. Similarly, $\nu_{2}\left(\nu_{3}\right)$ is the only $2 \nu_{2}<1$ $\left(2 \nu_{3}<1\right)$ eigenvalue from the partial transposition of the 2nd (3rd) mode.

We remind one more time that introduction of the noise-volume $\mu=2 \nu_{1} 2 \nu_{2} 2 \nu_{3}$ via the symplectic eigenvalues follows from the observation $\log _{2} 2 \nu_{-}=$ $\log _{2}\left(\lambda_{1, \mathrm{sm}}^{(\mathrm{in})} \lambda_{2, \mathrm{sm}}^{(\mathrm{in})}\right)$ for a single BS [17, 21, 22, where $2 \nu_{-}=$ $\lambda_{1, \mathrm{sm}}^{(\mathrm{in})} \lambda_{2, \mathrm{sm}}^{(\mathrm{in})}$ determines the maximum entanglement extractable from the input noise-area $\lambda_{1, \mathrm{sm}}^{(\mathrm{in})} \lambda_{2, \mathrm{sm}}^{(\mathrm{in})}$.

Calculation of $\boldsymbol{\eta}_{\text {ent }}$. - The nonclassical depth, associated only with the entanglement, is calculated as follows. We first transform the real noise-matrix $V$ into the complex representation 32 $V^{(\mathrm{c})}=\mathrm{CV}^{\dagger}$, where $\mathcal{C}=\left[\mathcal{C}_{1}, 0_{2 \times 2}, 0_{2 \times 2} ; 0_{2 \times 2}, \mathcal{C}_{1}, 0_{2 \times 2} ; \mathcal{C}_{1}, 0_{2 \times 2}, 0_{2 \times 2}\right]$ with $\mathcal{C}_{1}=[1, i ; 1,-i] / \sqrt{2}$. In the $6 \times 6$ complex noise-matrix $V^{(\mathrm{c})}$, we wipe out the SM nonclassicalities of the 3 modes, e.g. in the 1 st mode, by replacing the $\left[V_{11}, V_{12} ; V_{2,1}, V_{2,2}\right]$ with $[1 / 2,0 ; 0,1 / 2]$, the noise-matrix for vacuum or a coherent state [21]. We wipe out the SMNc of the other $2 \times 2$ block-diagonals similarly. Then, we obtain the new noise-matrix $V_{\text {ent }}^{(\mathrm{c})}$, where the nonclassical depths $\tau_{1,2,3}$ accounts the entanglement only. For Gaussian states, we consider here, this can be performed by calculating the $\tau_{1}, \tau_{2}, \tau_{3}$ which makes all eig $\left[\mathrm{V}_{\text {ent }}^{(\mathrm{c})}+\boldsymbol{\tau}\right]$ positive where $\boldsymbol{\tau}=\operatorname{diag}\left[\tau_{1}, \tau_{1}, \tau_{2}, \tau_{2}, \tau_{3}, \tau_{3}\right]$. Ref. [22] assigns a single $\tau_{1}=\tau_{2}=\tau_{3}=\tau$ for the $\tau$ matrix which certainly increases the injected noise. We quantify the nonclassicality of $V_{\text {ent }}^{(\mathrm{c})}$, or the entanglement, by choosing the minimum of $\tau_{\text {ent }}=\left[\tau_{1} \tau_{2} \tau_{3}\right]_{\text {min }}$, or $\eta_{\text {ent }}=$ $\left[\left(1-2 \tau_{1}\right)\left(1-2 \tau_{2}\right)\left(1-2 \tau_{3}\right)\right]_{\max }$ in terms in terms of the injected noise-volume. We note that, for a single-mode, $\lambda_{\mathrm{sm}}=(1-2 \tau)$ corresponds to the reduced noise of that particular mode.

Calculation of $\mathcal{N}_{\mathrm{SMNc}}$. - In the calculation of SMNc $\mathcal{N}_{\text {SMNc }}$, this time, we wipe out the correlations in the noise-matrix and left with the three $2 \times 2$ block-diagonals. $2 \times 2$ matrices give the $\mathrm{SMNc}$ associated with each mode [17]. Then, we introduce the SMNc noise-volume $\mathcal{N}_{\mathrm{SMNc}}=\left(1-2 \tau_{1}^{\text {(SMNc) }}\right)\left(1-2 \tau_{2}^{\text {(SMNc) }}\right)\left(1-2 \tau_{3}^{(\mathrm{SMNc})}\right)$.

\section{4-mode entanglement}

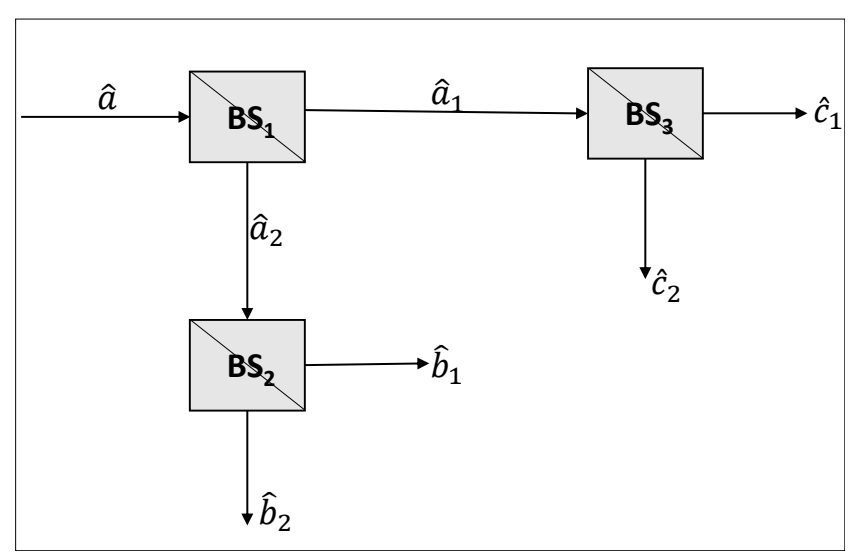

FIG. 3. Creation of 4-mode entanglement (4ME) with 3 beam-splitters. We examine the $4 \mathrm{ME}$ of $\hat{b}_{1}, \hat{b}_{2}, \hat{c}_{1}, \hat{c}_{2}$ modes. We also investigate the conversion of the single-mode nonclassicality into $4 \mathrm{ME}$, see Fig. 4

We perform similar calculations also for a 4-mode system given in Fig. 3. We obtain the same behavior depicted in Fig. 4 .

\section{Summary}

In summary, we introduce quantifications for 3-mode and 4-mode entanglement via two independent methods. We demonstrate how single-mode nonclasscality is converted into 3-mode and 4-mode entanglement. We quantify all nonclassicalities in terms of noise-volume. A

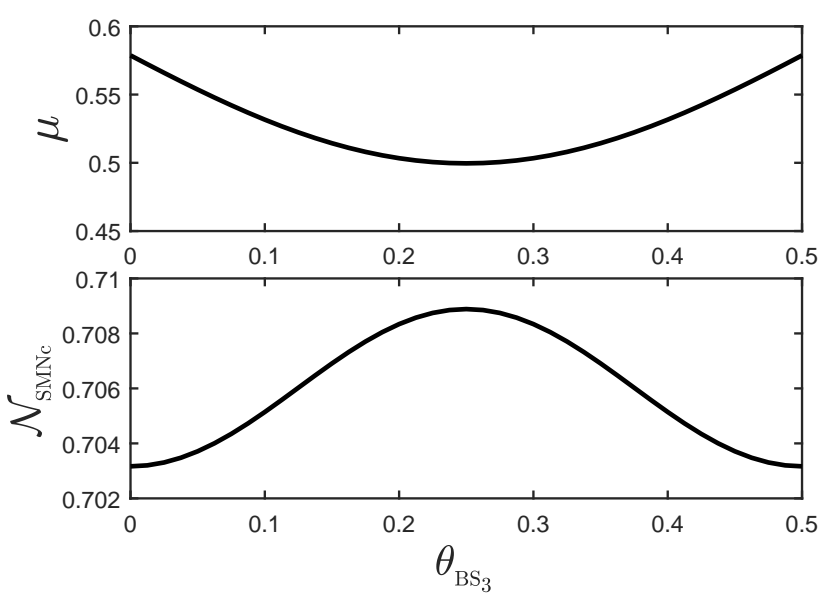

FIG. 4. The same of Fig. 2 for the 4-mode entanglement (4ME) for the system given in Fig. 3. We observe that SMNc is swapped into $4 \mathrm{ME}$. We cannot plot $\eta_{\text {ent }}$ since its calculation for a $4 \mathrm{D}$ system is seriously time-consuming. 
smaller noise-volume implies a stronger nonclassicality or entanglement. The method we introduce here can be generalized to other multi-mode entanglement which has fundamental importance in quantum communication.

\section{Acknowledgments}

MET acknowledges support from TUBITAK Grant
No: 1001-117F118, TUBA-GEBIP Award 2017, Hacettepe University BAP Grant No: FBI-2018-17423.
[1] Akira Furusawa and Peter van Loock, Quantum Teleportation and Entanglement: A Hybrid Approach to Optical Quantum Information ProcessingS (WILEY-VCH, 2011).

[2] Nicolas C Menicucci, Peter Van Loock, Mile Gu, Christian Weedbrook, Timothy C Ralph, and Michael A Nielsen, "Universal quantum computation with continuous-variable cluster states," Physical review letters 97, 110501 (2006).

[3] Robert Raussendorf and Hans J Briegel, "A one-way quantum computer," Physical Review Letters 86, 5188 (2001).

[4] Seiji Armstrong, Jean-François Morizur, Jiri Janousek, Boris Hage, Nicolas Treps, Ping Koy Lam, and Hans-A Bachor, "Programmable multimode quantum networks," Nature communications 3, 1026 (2012).

[5] H Jeff Kimble, "The quantum internet," Nature 453, 1023 (2008).

[6] Peter van Loock and Samuel L Braunstein, "Multipartite entanglement for continuous variables: a quantum teleportation network," Physical Review Letters 84, 3482 (2000).

[7] Mehmet Emre Tasgin, "Many-particle entanglement criterion for superradiantlike states," Physical review letters 119, 033601 (2017).

[8] J Hald, JL Sørensen, Christian Schori, and ES Polzik, "Spin squeezed atoms: a macroscopic entangled ensemble created by light," Physical Review Letters 83, 1319 (1999).

[9] Giuseppe Vitagliano, Giorgio Colangelo, F Martin Ciurana, Morgan W Mitchell, Robert J Sewell, and Géza Tóth, "Entanglement and extreme planar spin squeezing," Physical Review A 97, 020301 (2018).

[10] Julien Vidal, "Concurrence in collective models," Physical Review A 73, 062318 (2006).

[11] Masahiro Kitagawa and Masahito Ueda, "Squeezed spin states," Physical Review A 47, 5138 (1993).

[12] A Sørensen, L-M Duan, JI Cirac, and Peter Zoller, "Many-particle entanglement with bose-einstein condensates," Nature 409, 63-66 (2001).

[13] Bartosz Regula, Marco Piani, Marco Cianciaruso, Thomas R Bromley, Alexander Streltsov, and Gerardo Adesso, "Converting multilevel nonclassicality into genuine multipartite entanglement," New Journal of Physics (2018).

[14] Mehmet Emre Taşgın and Pierre Meystre, "Spin squeezing with coherent light via entanglement swapping," Physical Review A 83, 053848 (2011).

[15] M. S. Kim, W. Son, V. Bužek, and P. L. Knight, "Entanglement by a beam splitter: Nonclassicality as a prerequisite for entanglement," Phys. Rev. A 65, 032323 (2002).
[16] János K. Asbóth, John Calsamiglia, and Helmut Ritsch, "Computable measure of nonclassicality for light," Phys. Rev. Lett. 94, 173602 (2005).

[17] Wenchao Ge, Mehmet Emre Tasgin, and M Suhail Zubairy, "Conservation relation of nonclassicality and entanglement for gaussian states in a beam splitter," Physical Review A 92, 052328 (2015).

[18] Ievgen I Arkhipov, Jan Peřina Jr, Jiří Svozilík, and Adam Miranowicz, "Nonclassicality invariant of general two-mode gaussian states," Scientific reports 6, 26523 (2016).

[19] Ievgen I Arkhipov, Jan Peřina Jr, Jan Peřina, and Adam Miranowicz, "Interplay of nonclassicality and entanglement of two-mode gaussian fields generated in optical parametric processes," Physical Review A 94, 013807 (2016).

[20] Antonín Černoch, Karol Bartkiewicz, Karel Lemr, and Jan Soubusta, "Experimental tests of coherence and entanglement conservation under unitary evolutions," Physical Review A 97, 042305 (2018).

[21] Rabia Tahira, Manzoor Ikram, Hyunchul Nha, and M. Suhail Zubairy, "Entanglement of gaussian states using a beam splitter," Phys. Rev. A 79, 023816 (2009).

[22] Hong-Rong Li, Fu-Li Li, and Yang Yang, "Entangling two single-mode gaussian states by use of a beam splitter," Chinese Physics 15, 2947 (2006).

[23] Gerardo Adesso, Alessio Serafini, and Fabrizio Illuminati, "Extremal entanglement and mixedness in continuous variable systems," Phys. Rev. A 70, 022318 (2004).

[24] G. Vidal and R. F. Werner, Phys. Rev. A 65, 032314 (2002).

[25] Mehmet Emre Tasgin, "Anatomy of entanglement and nonclassicality criteria," arXiv preprint arXiv:1901.04045 (2019).

[26] Lu-Ming Duan, G. Giedke, J. Cirac, and P. Zoller, "Inseparability criterion for continuous variable systems," Phys. Rev. Lett. 84, 2722-2725 (2000)

[27] Mark Hillery and M. Zubairy, "Entanglement conditions for two-mode states," Phys. Rev. Lett. 96, 050503 (2006).

[28] Ching Tsung Lee, "Measure of the nonclassicality of nonclassical states," Physical Review A 44, R2775 (1991).

[29] Martin B Plenio, "Logarithmic negativity: A full entanglement monotone that is not convex," Phys. Rev. Lett. 95, 090503 (2005).

[30] T Kiesel and W Vogel, "Nonclassicality filters and quasiprobabilities," Physical Review A 82, 032107 (2010).

[31] M. O. Scully and M. S. Zubairy, Quantum Optics (Cambridge University Press, New York, 1997).

[32] R Simon, N Mukunda, and Biswadeb Dutta, "Quantumnoise matrix for multimode systems: $U(n)$ invariance, 
squeezing, and normal forms," Physical Review A 49, 1567 (1994).

[33] R. Simon, "Peres-horodecki separability criterion for con- tinuous variable systems," Phys. Rev. Lett. 84, 2726$2729(2000)$. 